\title{
EFEITO DA SUBMERSÃO EM ÁGUA DE SEMENTES DE FEIJÃO NA GERMINAÇÃO E NO VIGOR ${ }^{1}$
}

\author{
CECI CASTILHO CUSTÓDIO², NELSON BARBOSA MACHADO NET O ${ }^{2}$, HÉLIO MASSAKI ITO³ ${ }^{3}$ MÁRCIA REGINA VIV AN ${ }^{4}$
}

\begin{abstract}
RESUMO - Este trabalho foi conduzido no Laboratório de Análise de Sementes da Faculdade de Ciências Agrárias da Universidade Oeste Paulista (UNOESTE), Presidente Prudente-SP, com o objetivo de avaliar os efeitos causados pelo alagamento na germinação e no vigor de sementes de feijão. As sementes foram submetidas aos tratamentos de alagamento por períodos de $0,8,16,24$, 32,40 e 48 horas a $25^{\circ} \mathrm{C}$. Durante os tratamentos foram realizadas as avaliações de grau de umidade inicial e final, e após os mesmos, teste de germinação e avaliação do comprimento de hipocótilo e de raiz, peso seco da parte aérea e raiz . Os resultados mostraram que a germinação e o vigor das sementes decresceram com o alagamento, havendo decréscimo médio de 55 pontos percentuais na germinação, com 8 horas de alagamento. Ocorreu diferenciação de lotes através da germinação com sementes que passaram por períodos de alagamento iguais ou superiores a 8 horas. O número de sementes mortas e a inibição ao desenvolvimento de raiz e hipocótilo foram crescentes com o aumento do período de submersão. Desta forma, concluiu-se que o alagamento, por 8 horas, pode causar prejuízos irreversíveis ao estabelecimento da cultura do feijoeiro, bem como, se empregado em laboratório, pode ser um bom indicativo para diferenciação de níveis de qualidade fisiológica em sementes de feijão.
\end{abstract}

Termos para indexação: alagamento, sementes, Phaseolus vulgaris L.

\section{THE FLOODING EFFECTS ON THE BEAN SEED GERMINATION AND VIGOR OF BEAN SEEDS}

\begin{abstract}
This work study was carried out to evaluate the effects of different flooding periods during germination and in the bean seeds vigor at UNOESTE Seed Analysis Laboratory. Seeds were submitted to flooding periods of $0,8,16,24,32,40$ and $48 \mathrm{~h}$ at $25^{\circ} \mathrm{C}$. Moisture content was taken before and after flooding periods. The germination test and physiological parameters, such as hypocotyl and root length and their dry weights, were obtained after periods of flooding. Results showed that germination and vigor decreased subsequently to flooding, germination decreased 55 percentage points after $8 \mathrm{~h}$ of seed submersion. There were differentiation differences between among seed lots in germination tests after flooding periods equal to, or higher than $8 \mathrm{~h}$. Dead seed number and inhibition in hypocotyl and root development, shown in this work study, increased with the submersion time. In conclusion, eight hours of flooding cause irreversible damage to bean culture establishment, and the same periods of submersion, in the laboratory, could be a very good indicative to differentiate seed physiological quality.
\end{abstract}

Index terms: flooding, seeds, Phaseolus vulgaris.

\footnotetext{
${ }^{1}$ Aceito para publicação em 20.10.2002; trabalho desenvolvido, pelos acadêmicos, como requisito para conclusão do curso de graduação em Engenharia Agronômica, Faculdade de Ciências Agrárias, Universidade do Oeste Paulista; Rodovia Raposo Tavares, Km 572, 19067175, Presidente Prudente-SP.

2 Prof., Dr. Departamento de Biologia Vegetal e Fitossanidade, UNOESTE; e-mail: ceci@ apecnt.unoeste.br

${ }^{3}$ Graduandos do Curso de Engenharia Agronômica, UNOESTE.
}

\section{INTRODUÇÃO}

O processo germinativo, em sementes, é dependente de vários fatores. $\mathrm{O}$ suprimento limitado de oxigênio para as sementes, durante o período de embebição, induz uma alteração da via respiratória aeróbia para a fermentativa ou anaeróbia (Neumann et al., 1999). Se, a via fermentativa seguida, for a etanólica, o produto desta poderá levar a um decréscimo no 
poder germinativo. Vários autores tem demonstrado a diminuição da germinação de várias espécies, quando submetidas ao alagamento, entre eles: Saka \& Izawa, 1999 (em arroz), Neumann et al., 1999 (em feijoeiro comum) e Narimanov \& Korystov, 1998 (em cevada).

Durante o alagamento do substrato ocorre, também, injúria por rápida embebição, isto é, entrada de água, em grande quantidade e rapidez, no interior da semente devido à diferença de potencial hídrico entre o interior da semente e o meio no qual ela se encontra, podendo aumentar, na fase de embebição, de -400MPa até -1MPa, o que reflete uma considerável entrada de água (Coll et al., 1992; Castro \& Vieira, 2001). O dano por embebição, portanto, será proporcional à diferença de potencial hídrico entre a semente e o meio. Desta forma, a semente, já danificada, tem menor quantidade de energia disponível para o processo germinativo, refletindo em menor vigor (Richard et al., 1991). No entanto, algumas sementes podem suportar inundações temporárias (Souza et al., 1999) e podem existir diferenças varietais na taxa de sobrevivência das sementes após o alagamento (Saka \& Izawa, 1999; Lenssen et al.,1999).

Em regiões úmidas e quentes, vários fatores bióticos e abióticos influenciam na perda de viabilidade e do vigor das sementes. Entre esses, o excesso de chuva, logo após a semeadura, pode ser um fator limitante à produção, por causar deterioração das sementes levando à menor emergência das plântulas em campo (Duke \& Kakefuda, 1981; Blom, 1999).

Este estudo teve como objetivo verificar o efeito de diferentes períodos de alagamento sobre a germinação e o vigor de sementes de feijão.

\section{MATERIAL E MÉTODOS}

O trabalho foi conduzido no Laboratório de Análise de Sementes da Faculdade de Ciências Agrárias da Universidade do Oeste Paulista (UNOESTE), Presidente Prudente-SP, nos meses de setembro e outubro de 2001.

Foram usadas, neste experimento, sementes de dois lotes de feijão adquiridas da empresa Campos Sales, cultivar Pérola, safra 2000/2001. As sementes foram submersas em $100 \mathrm{ml}$ de água destilada em copos plásticos de $250 \mathrm{ml}$, contendo, cada copo, 100 sementes, sendo utilizados 4 copos por tratamento. Para evitar a proliferação de fungos e bactérias foram adicionados, na solução de alagamento, fungicida (Carboxin $200 \mathrm{~g} / \mathrm{l}+$ Thiram $200 \mathrm{~g} / \mathrm{l}$ ) na dose de $0,2 \%$ de produto comercial e ainda um bactericida e fungicida, KILOL? na proporção de 1:125 (v:v). As sementes submetidas ao ala- gamento foram, então, incubadas, em germinador, a temperatura de $25^{\circ} \mathrm{C}$, no escuro, por $0,8,16,24,32,40$ e 48 horas. Após os períodos de alagamento, as sementes foram submetidas ao teste de germinação, de acordo com as Regras para Análise de Sementes (Brasil, 1992). As quatro subamostras de 50 sementes de cada tratamento foram distribuídas sobre 2 folhas de papel toalha para germinação, umedecidas com quantidade de água equivalente a 2,5 vezes o peso do substrato, cobertas com 1 folha e, após, confeccionados rolos de germinação que foram mantidos em germinador a $25^{\circ} \mathrm{C}$. As avaliações foram realizadas aos 5 e 8 dias após a semeadura e os resultado expressos em porcentagem média de plântulas normais, plântulas anormais e de sementes mortas, conforme indicado em Brasil (1992).

A avaliação do crescimento de plântulas foi realizada de acordo com Nakagawa (1999). Foram distribuídas 10 sementes sobre uma linha traçada no terço superior, no sentido longitudinal do papel substrato pré-umedecido. Foram confeccionados rolos semelhantes ao teste de germinação (Brasil, 1992) produzindo quatro repetições por tratamento que foram levadas ao germinador, por 5 dias, seguindo-se os procedimentos descritos para o teste de germinação. As plântulas normais obtidas foram avaliadas por comprimento do hipocótilo e da raiz primária. Após as medições, as mesmas foram seccionadas obtendo-se a parte aérea, desprezando-se os cotilédones, e a raiz. Em seguida foram acondicionadas em sacos de papel e levadas à estufa com circulação de ar a $60^{\circ} \mathrm{C}$, até atingir peso constante, para avaliação do peso seco de parte aérea e de raiz.

O delineamento experimental utilizado foi o inteiramente casualizado com 4 repetições. Os dados porcentuais de plântulas normais, anormais e de sementes mortas foram transformados em arco-seno da raiz quadrada de $\mathrm{x} / 100$ enquanto os resultados de comprimento e peso não foram transformados. A análise de variância foi realizada aplicando-se o teste $\mathrm{F}$, e quando este foi significativo, a comparação de médias foi feita através do teste Tukey a 5\% de significância, para comparação de lotes, e através de análise de regressão poli-nomial, para comparação dos níveis de alagamento. Para a análise estatística dos dados utilizou-se o software SANEST.

\section{RESULTADOS E DISCUSSÃO}

Os resultados de determinação do grau de umidade das sementes, antes e após terem sido submetidas aos tratamentos de alagamento (Tabela 1) mostraram que o teor de água 
TABELA 1. Médias de determinação do grau de umidade nas sementes de feijão antes e após os tratamentos de alagamento. Presidente Prudente, SP, 2001.

\begin{tabular}{lrc}
\hline \multicolumn{1}{c}{ Tratamento } & Lote A & Lote B \\
& $\ldots \ldots \ldots \ldots \ldots \ldots(\%)$ & $\ldots \ldots \ldots \ldots \ldots \ldots . . . \ldots \ldots$ \\
\hline 0 horas (sem alagamento) & 9,9 & 11,3 \\
8 horas & 52,4 & 51,3 \\
16 horas & 57,1 & 57,5 \\
24 horas & 57,7 & 58,7 \\
32 horas & 57,5 & 57,3 \\
40 horas & 57,4 & 58,4 \\
48 horas & 57,7 & 59,5
\end{tabular}

passou de 9,9 e 11,3\% na semente quiescente para 52,4 e $51,3 \%$ após 8 horas de alagamento, respectivamente para os lotes A e B, estabilizando-se com valores próximos a 58\%, indicando que o processo de germinação foi comprometido em função do alagamento (Figura 1).

Os resultados da análise de variância (Tabela 2) indicaram que as variáveis germinação (plântulas normais), plântulas anormais e sementes mortas apresentaram médias que diferiram significativamente entre os períodos de alagamento e entre os dois lotes estudados. No entanto, os comprimentos de plântulas (raiz e hipocótilo) e os pesos secos (de raiz e de

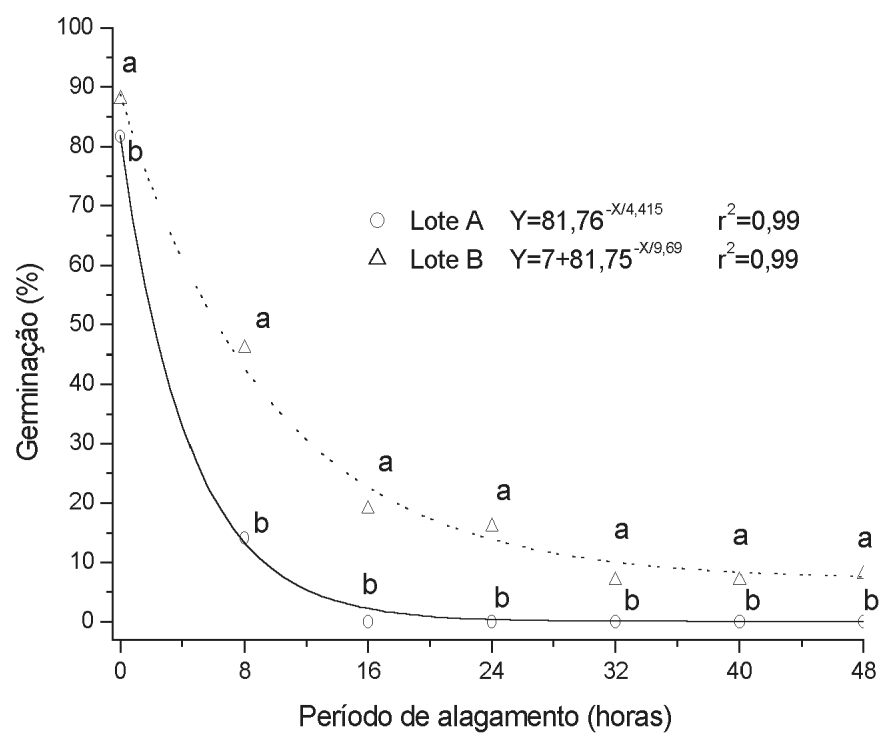

FIG. 1. Resultados médios de germinação (\%), de dois lotes de sementes de feijão, obtidos através de diferentes períodos de alagamento. Presidente Prudente, SP, 2001.
TABELA 2. Resultados obtidos na análise de variância, pelo teste $\mathrm{F}$, para os tratamentos de alagamento, lotes e para a interação tratamentos e lotes em plântulas normais (PN), anormais (PA) e sementes mortas (SM), comprimento de raiz $(\mathrm{CR})$ e de hipocótilo $(\mathrm{CH})$, peso seco de raiz (PSR) e de parte aérea (PSPA). Presidente Prudente, SP, 2001.

\begin{tabular}{lccccccc}
\hline & PN & PA & SM & CR & CH & PSR & PSPA \\
\hline Tratamentos (T) & $* *$ & $* *$ & $* *$ & $* *$ & $* *$ & $* *$ & $* *$ \\
Lotes (L) & $* *$ & $* *$ & $* *$ & n.s. & n.s. & n.s. & n.s. \\
$\mathrm{T} * \mathrm{~L}$ & $*$ & $* *$ & $* *$ & n.s. & n.s. & n.s. & n.s.
\end{tabular}

** significativo a $1 \%$; * significativo a $5 \%$; n.s. não significativo

parte aérea) apresentaram médias que diferiram significativamente entre os períodos de alagamento, porém ambos os lotes comportaram-se de modo semelhante.

Os resultados de germinação e de vigor das sementes foram reduzidos logo após as mesmas serem submetidas ao alagamento por $8,16,24,32,40$ e 48 horas, como mostram as Figuras 1, 4 e 5. Após 8 horas de alagamento, ocorreu queda na germinação e no vigor, com significativo acréscimo de plântulas anormais (Figura 2) e no número de sementes mortas (Figura 3).

Os resultados médios de plântulas normais (Figura 1) revelaram que as sementes que não foram submetidas ao ala-

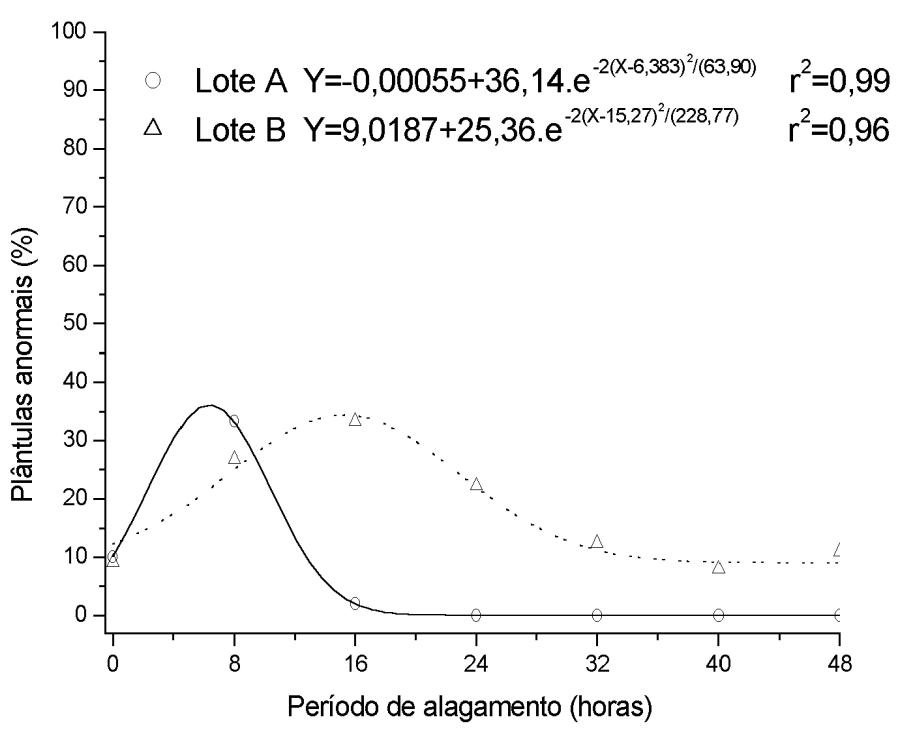

FIG. 2. Resultados médios de plântulas anormais (\%), de dois lotes de sementes de feijão, obtidos através de diferentes períodos de alagamento. Presidente Prudente, SP, 2001. 
gamento produziram cerca de 82 e $88 \%$ de plântulas normais e as que foram, por 8 horas, 14 e $46 \%$, respectivamente para os lotes A e B. Nos demais períodos de alagamento (16, 24, 32, 40 e 48 horas), a germinação das sementes foi severamente reduzida. Isto mostra que as sementes de feijão não suportam alagamento, devido a falta de oxigênio, e acabam morrendo. Oito horas de alagamento foram suficientes para

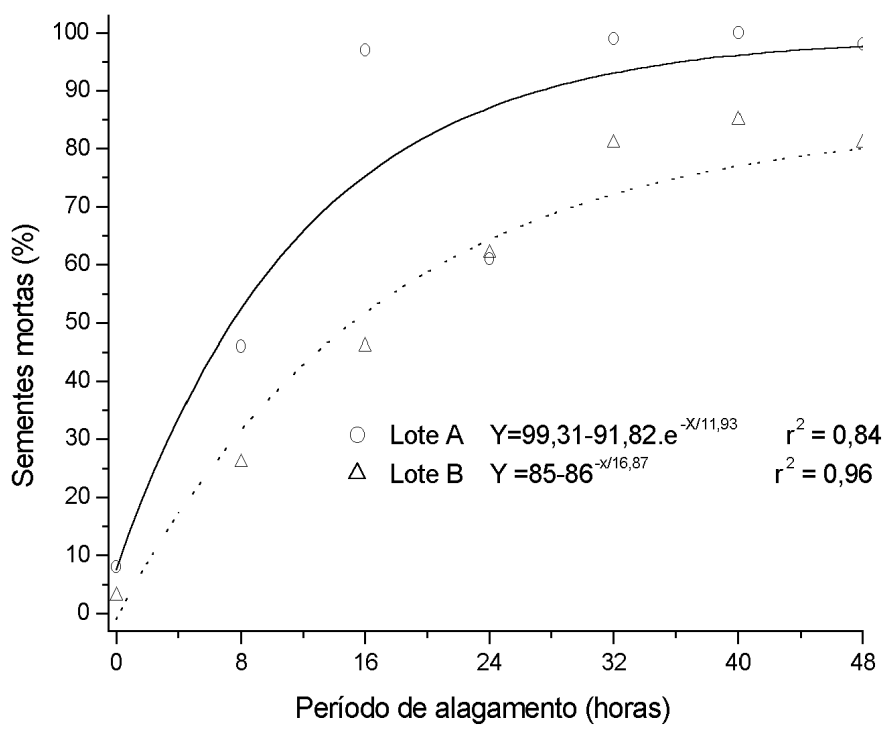

FIG. 3. Resultados médios de sementes mortas (\%), de dois lotes de sementes de feijão, obtidos através de diferentes períodos de alagamento. Presidente Prudente, SP, 2001.

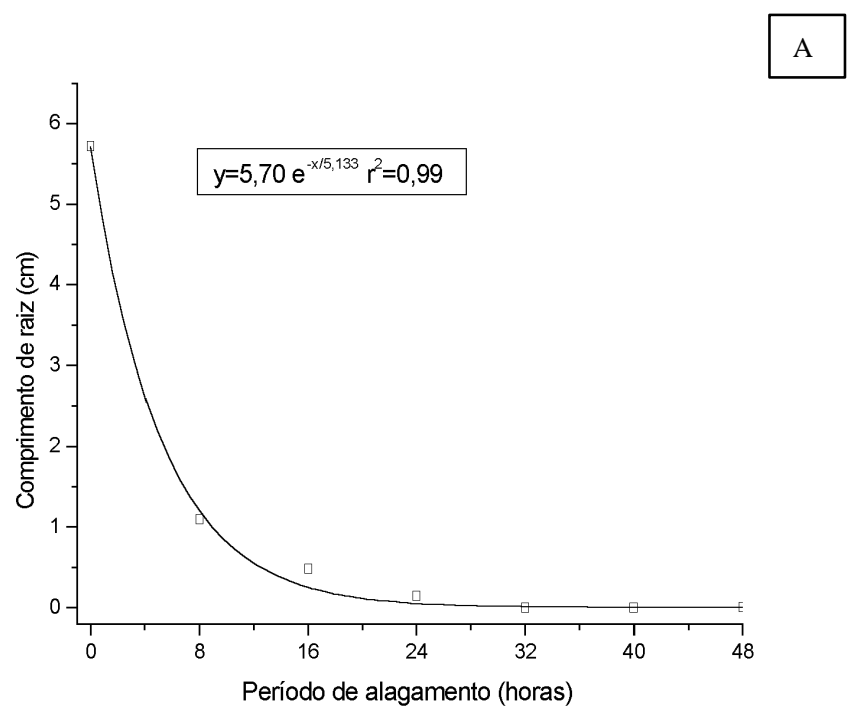

causar decréscimo na germinação em aproximadamente 68 e 42 pontos percentuais, respectivamente para os lotes A e B. Esta situação pode ocorrer em campo, principalmente no verão, quando a intensidade e duração das chuvas são maiores (Dennis et al., 2000). Segundo Naseem et al. (1997), em situação de campo, os maiores índices de estabelecimento de plântulas de feijão ocorreram com umidade do solo próxima a $40-45 \%$. Valores superiores e inferiores acarretaram prejuízos ao estabelecimento de populações adequadas de plantas por causarem diminuição da germinação das sementes.

$\mathrm{Na}$ Figura 2 as sementes que não foram submetidas ao alagamento, apresentaram apenas 10 e $9 \%$ de plântulas anormais, respectivamente para os lotes $\mathrm{A}$ e $\mathrm{B}$, enquanto as que passaram por 8 horas de alagamento, exibiram 33 e $27 \%$ de plântulas anormais, respectivamente. Para os demais períodos de alagamento, a porcentagem de plântulas anormais foi menor, devido a porcentagem de germinação ter sido praticamente nula. Isto significa que as sementes que passam por submersão, tenderam a apresentar porcentagem de plântulas anormais maiores em relação às sementes não alagadas, porém quando o alagamento foi prolongado, ocorreu acentuada morte de sementes (Figura 3). No entanto, algumas sementes podem suportar inundações temporárias (Souza et al., 1999) e pode existir diferenças varietais na taxa de sobrevivência das sementes após alagamento (Saka \& Izawa, 1999; Lenssen et al., 1999).

$\mathrm{Na}$ Figura 3 foram apresentados os resultados porcentuais de sementes mortas após submersão. Aquelas que passaram 8 horas submersas apresentaram 46 e $26 \%$ de se-

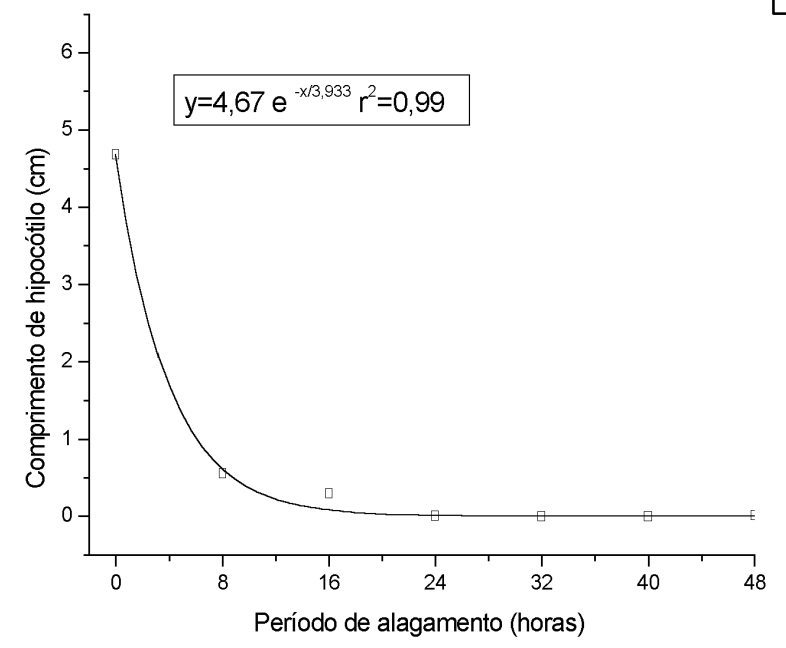

FIG. 4. Resultados médios de comprimento de raiz (A) e de hipocótilo (B), de sementes de feijão, obtidos através de diferentes períodos de alagamento. Presidente Prudente, SP, 2001. 

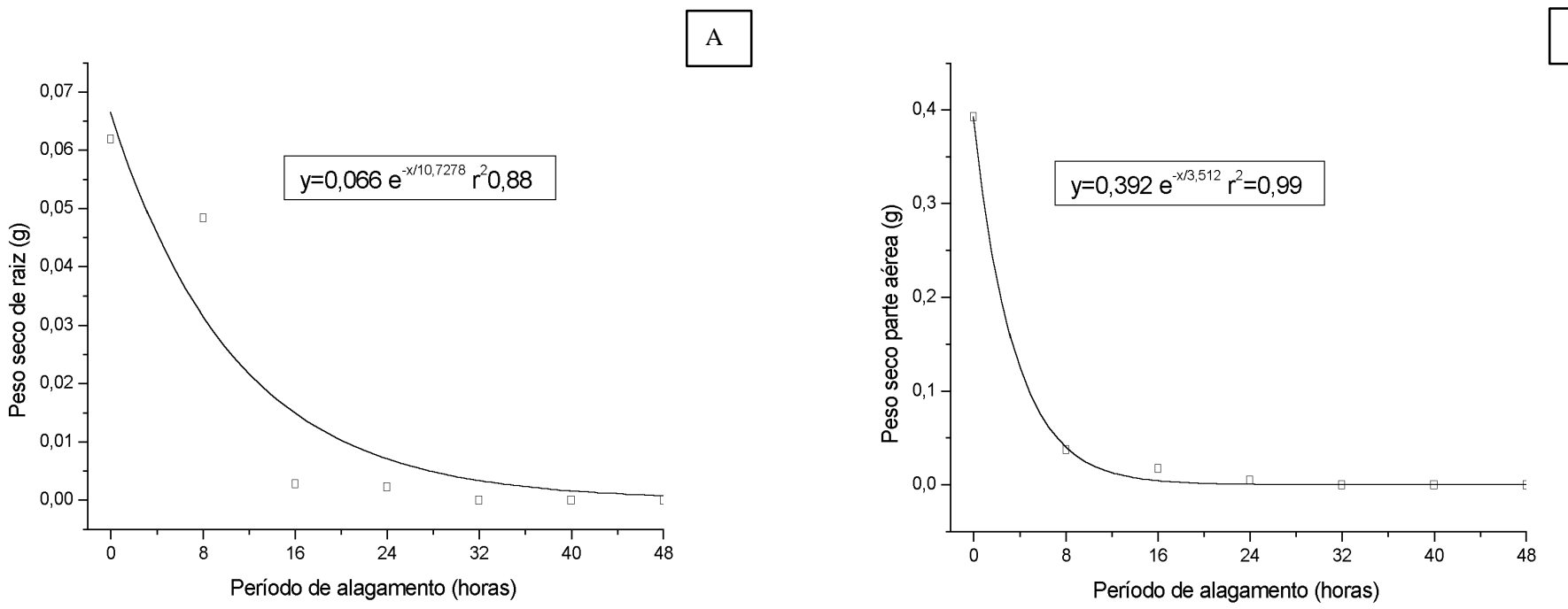

FIG. 5. Resultados médios de peso seco de raiz (A) e de parte aérea (B), de sementes de feijão, obtidos através de diferentes períodos de alagamento. Presidente Prudente, SP, 2001.

mentes mortas, respectivamente, para os lotes A e B, enquanto as que não sofreram alagamento, 8 e $3 \%$ de sementes mortas, respectivamente, ou seja, o alagamento, por 8 horas, foi suficientemente drástico a ponto de aumentar a incidência de sementes mortas em 38 e 23 pontos percentuais. As quantidades de sementes mortas atingiram valores de $100 \mathrm{e}$ $81 \%$ com 32 horas de submersão. Estes resultados indicaram que as sementes de feijão são sensíveis às condições de alagamento. Por outro lado, Dantas et al.(2000) relataram que ocorreu decréscimo na germinação, de 40 pontos percentuais, após 3 dias, trabalhando com milho, e que a germinação foi nula após 7 dias de submersão.

A morte de plantas e sementes podem ser atribuídas aos produtos tóxicos do metabolismo anaeróbio como acetaldeído e etanol (Crawford, 1978). $\mathrm{O} \mathrm{CO}_{2}$ liberado no meio de inundação também pode inibir a germinação (Narimanov \& Korystov, 1998). Todavia, segundo Martin et al. (1991) e van Toai et al. (1988) não há inibição da germinação devido ao acúmulo destas substância, que ocorreriam em concentrações insuficientes para provocar fitotoxicidade. Algumas enzimas, no entanto, podem estar relacionadas com a inibição da germinação e/ou crescimento de raiz primária em situações de anóxia e hipóxia como invertase e sucrose sintase (Zeng et al., 1999) e alcool desidrogenase (Chung \& Ferl, 1999).

Os resultados de comprimento de raiz e de hipocótilo (Figura 4) e de peso seco de raiz e de parte aérea (Figura 5) apresentaram redução em todos os períodos de alagamento. O comportamento dos lotes A e B foi semelhante de tal maneira que, para os parâmetros indicados no início do parágra- fo, não houve significância, para lotes, na análise de variância (Tabela 2).

\section{CONCLUSÃO}

$£ \mathrm{O}$ vigor e a germinação de sementes de feijão são afetados negativamente pelo alagamento;

exoito horas de alagamento podem provocar prejuízos irreversíveis ao estabelecimento da cultura;

\&a submersão das sementes em água, se empregada em laboratório, pode ser um bom indicativo para diferenciação de níveis de qualidade fisiológica em sementes de feijão.

\section{REFERÊNCIAS}

BLOM, C.W.P.M. Adaptations to flooding stress:from plant community to molecule. Plant Biology, Stuttgart, v.1, n.3, p.261273, 1999.

BRASIL. Ministério da Agricultura e Reforma Agrária. Regras para análise de sementes. Brasília:SNAD/DNDV/CLAV, 1992. 365p.

CASTRO, P.R.C.; VIEIRA, E.L. Aplicações de reguladores vegetais na agricultura tropical. Guaíba: Livraria e Editora Agropecuária, 2001. 132p.

CHUNG, H.; FERL, R.J. Arabidopsis alcool dehydrogenase expression in both shoots and roots is conditioned by root growth environment. Plant Physiology, Rockville, v.121, n.2, p.429-436.

COLL, J.B.; RODRIGO, G.N.; GARCIA, B.S.; TAMÉS, R.S. Fisiologia vegetal. Madrid: Piramide, 1992. 662p.

CRAWFORD, R.M.M. Metabolic adaptations to anoxia. In: HOOK, 
D.D.; CRAWFORD, R.M.(Ed.). Plant life in anaerobic environments. Ann Arbor: Ann Arbor Science, 1978. p.119-136

DANTAS, B.F.; ARAGÃO, C.A.; CAVARIANI, C.; NAKAGAWA, J.; RODRIGUES, J.D. Efeito da duração e da temperatura de alagamento na germinação e no vigor de sementes de milho. Revista Brasileira de Sementes, Brasília, v.22, n.1, p.88-96, 2000.

DENNIS, E.S.; DOLFERUS, R.; ELLIS, M.; RAHMAN, M.; WU, Y.; HOEREN, F.U.; GROVER, A.; ISMOND, K.P.; GOOD, A.G.; PEACOCK, W.J.; PARRY, M.; FOYER, C.; FORDE, B. Molecular physiology: engineering crops for hostile environments. Journalof Experimental Botany, Oxford, v.51, n.342, p.89-97, 2000.

DUKE, S.H.; KAKEFUDA, G. Role of testa in preventing cellular rupture during imbibition of legume seeds. Plant Physiology, Rockville,v.67,n.3,p.449-456,1981.

LENSSEN, J.P.M.; MENTING, F.B.J.; PUTTEN, W.; BLOM, K. Control of plant species richness and zonation of functional groups along a freshwater flooding gradient. Oikos, Lund, v.86, n.3, p.523534, 1999.

MARTIN, B.A.; CERWICK, S.F.; REDING, L.D. Physiological basis for inhibition of maize seed germination by flooding. Crop Science, Madison, v.31, n.6, p.152-157, 1991.

NAKAGAWA, J. Testes de vigor baseados no desempenho das plântulas. In: KRZYZANOWSKI, F.C., VIEIRA, R.D., FRANÇA NETO, J.B. (Ed.). Vigor de sementes: conceitos e testes. Londrina: ABRATES, 1999. 218p.

NARIMANOV, A.A.; KORYSTOV, Y.N. The mechanism responsible for the increaes of barley seed germination rate in the presence of hydrogen peroxide after excessive wetting. Biology Bulletin of the Russian Academy of Sciences, Moscow, v.25, n.1, p.93-96, 1998.
NASEEM, S.B.; KHAN, A.H.; ISLAM, M.; MOLLAH, U.; ALI, M.A. Effect of seeding methods and varying surface soil moisture on the stand establishment of mungbean (Vigna radiata L.). Bangladesh Journal of Scientific and Industrial Research, Dacca, v.32, n.2, p.295-301, 1997.

NEUMANN, G.; PREISSLER, M.; AZAIZEH, H.A.; ROMHELD, $\mathrm{V}$. Thiamine (vitamin B1) deficiency in germinating seeds of Phaseolus vulgaris L. exposed to soaking injury. Journal of Plant Nutrition and Soil Science, Weinheim, v.162, n.3, p.295-300, 1999.

RICHARD, B., RIVOAL, J.; SPITERI, A., PRADET, A. Anaerobic stress induces the transcription and translation of sucrose synthase in rice. Plant Physiology, Rockville, v.95, n.3, p.669674, 1991.

SAKA, N., IZAWA, T. Varietal differences in the survival rate of sprouting rice ssed (Oryza sativa L.) under highly reduced soil conditions. Plant Production Science, Tokyo, v.2, n.2, p.136-137, 1999.

SOUZA, A.F.; ANDRADE, A.C.S.; RAMOS, F.N.; LOUREIRO, M.B. Ecophysiology and morphology of seed germination of the neotropical lowland tree Genipa americana (Rubiaceae). Journal of Tropical Ecology, Cambridge, v.15, p.667-680, 1999.

TOAI, T.T. van; FAUSEY, N.R.; McDONALD JUNIOR, M.B. Oxygen requirements for germination and growth of floodsusceptible and flood-tolerant corn lines. Crop Science, Madison, v. 28, n. 5, p. 79-83, 1988.

ZENG, Y.; WU, Y.; AVIGNE, W.T.; KOCH, K.E. Rapid repression of maize invertases by low oxygen: invertase/sucrose synthase balance, sugar signalling potential, and seedling survival. Plant Physiology, Rockville, v.121, n.2, p.599-608. 